\title{
Supporting situational awareness through a patient overview screen for Bipolar Disorder treatment
}

\author{
Mads Frost \\ Pervasive Interaction Technology Lab, \\ IT University of Copenhagen, \\ Copenhagen, Denmark \\ madsf@itu.dk
}

\begin{abstract}
Situational awareness, and how systems can be designed to support it, has been a focus in many dynamic, safety critical contexts, with great success. The work presented here extends the study of situation awareness into the domain of patient overview screens in personal monitoring systems. In this paper we report on the design and formative evaluation of a detailed patient overview screen for supporting the treatment of bipolar disorder through MONARCA, a personal monitoring system. We define the key items for supporting situational awareness for clinicians, as well as discuss key findings such as doctors vs. nurses needs and the lack of need for situational awareness support.
\end{abstract}

Keywords - healthcare, mental illness, situational awareness, bipolar disorder, personal monitoring system, overview screen, user centered design, usability evaluation.

\section{INTRODUCTION}

Bipolar disorder is associated with a high risk of relapse and hospitalization [1]. Major reasons for the decreased effect of interventions in clinical practice are delayed intervention for depressive and manic episodes [2, 3]. Recently, electronic solutions for self-monitoring of affective symptoms using cell phones have been suggested as an easy and cheap way to identify early signs of affective episodes $[4,5]$.

As the adoption of information technology has increased, so too has the demand that these systems adapt to the clinical environment, and make accessing and managing information easier [6]. With the introduction of personal monitoring systems for conditions like diabetes [7, 8], chronic kidney disease [9], and asthma [10], the amount of data to be interpreted by clinicians has increased.

In particular, clinicians should be supported in achieving a good level of Situational Awareness (SA) about their patient's condition at the point of care, when decisions need to be taken. SA refers to the capacity of "knowing what is going on around you to decide what to do" [11] and is a field of study concerned with understanding how perception, comprehension and projection processes can be supported in order to enable effective decision-making in complex, dynamic domains [12]. These are domains where the information flow is quite high and poor decisions may lead to serious consequences (e.g., air traffic control, emergency response and healthcare management). Situational awareness is a state achieved when information that is qualitatively and quantitatively relevant for the decision-maker is made available through appropriate systems and information exchange patterns [13].

\author{
Silvia Gabrielli \\ Ubiquitous Interaction Group \\ CREATE-NET \\ Trento, Italy \\ silvia.gabrielli@create-net.org
}

Also, previous work in the field of Computerized Decision Support Systems has shown that health information technology components can positively impact chronic illness care [14]. Aspects of health IT systems found to be correlated with enhanced health outcome included, among the others, linkage between the technology system and an electronic medical record, computerized prompts during treatment decision-making, availability of progress reports and feedback, specialized decision support [15]. These studies have also pointed out the importance of providing clinician feedback at the time of the patient visit, in order to support the quality of their decision-making and their assessment of symptoms and side effects at the point of care [15]. Although health IT systems like EMRs can facilitate within-office care coordination, by providing access to data during patient encounters, it has also been shown that: (i) EMRs are less able to support coordination between clinicians and settings, in part due to their design and a lack of standardization of key data elements required for information exchange, and (ii) managing information overflow from EMRs is a challenge for clinicians [16]. Moreover, how medical information is presented (its context) is an important factor affecting data retrieval and interpretation by clinicians. Computer systems should be capable of producing well-structured information screens, based on relevant objective and subjective clinical data, so as to improve retrieval and assimilation of existing information on patients, improve comprehension and provide a more holistic view of the patient [17]. Usability is thus critical to successful health IT implementation and adoption and its subsequent ability to improve health care quality.

During the first field trial of the MONARCA SelfAssessment System, we found the need for a more effective design of overview interfaces, supporting the healthcare personnel involved in the treatment of bipolar patients. In particular, the need for supporting the SA at the point of care of doctors and nurses, by presenting them with the most relevant monitored data, provided through the use of the MONARCA system, and additional clinical data regarding the patients, which could inform clinicians' decision-making more effectively at the current point in treatment. Previously in the system, clinicians went from high-level overview of all patients directly into detailed data regarding the individual patient, leaving the clinician to rummage through vast amounts of data in order to get an overview of the patient. Therefore there was a need for an intermediate layer of 
information, consisting of an overview of the individual patient and the relevant information.

In this paper, we first describe how we approached the challenge of designing the detailed patient overview screen, the formative evaluations conducted with usability experts as well as clinical staff. We conclude with a discussion of the outcome and lessons learned from these evaluations.

\section{DESIGN PROCESS}

Designing for bipolar disorder poses several challenges. Due to its complexity, it is unclear what data are most important. Symptoms vary from patient to patient, and may be difficult to recognize. Thus, the design of the patient overview screen was done in a series of user-centered design workshops, involving three doctors and a nurse affiliated with the psychiatric clinic of a large university hospital in Denmark.

The initial idea was to design an intelligent user interface, which could be tailored to the needs of individual patients, following practical guidelines for treatment [18]. However, when this idea was presented to the clinicians in the workshop, they didn't see the need for an adaptive system - they much preferred a unified system, which always displayed the same key information they were interested in when treating the patient.

Long discussions were undertaken on perspectives of the treatment, different categories and items needed for providing the best possible overview of the patient, drawing from their respective best practices: both medically and practically. In this stage, it became evident that doctors and nurses have different needs and requirements based on their work with the patients, which is discussed in further detail in the discussion section of the paper. However, we did manage to find a solution, which meets both parties' needs. It contains the data needed for both parties to preform their work, but at the same time also makes it easier when collaborating on treating the patient, with a common reference point.

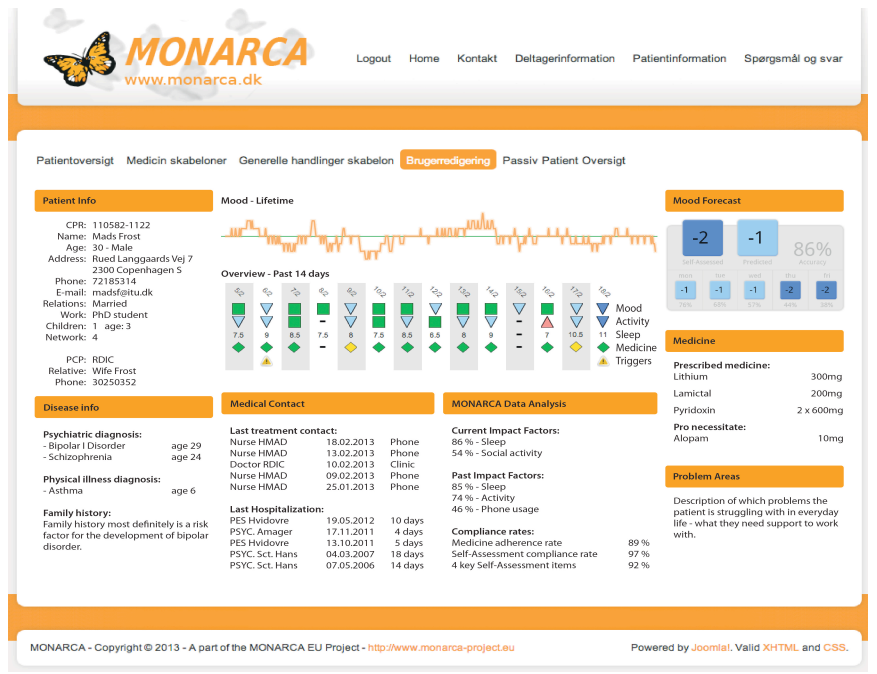

Figure 1: Patient overview screen mock-up, as used in the evaluation.

We provided the participants with materials such as large poster paper, writing materials, scissors, tape, etc. The sketches that came out of this initial brainstorming formed the basis for the first mockup. The mockup was then computerized, and discussed again, to provide the final evaluation version, as seen in Fig. 1.

\section{PATIENT OVERVIEW SCREEN}

The overview screen, as seen in Fig. 1, consists of 9 main categories. On the left side from the top;

- Patient info - containing Social security number, Name, Age, Address, Phone, E-mail, Relationship status, Work, Number and age of children, Network score on a 1-5 scale; the higher the number, the better help and support they have from family and friends, PCP - Primary Care Providers - the clinicians in charge of the patient's care, Name of Relative, and a contact number for the relative.

- Disease info - containing the patient's psychiatric and physical diagnoses, according to ICD-10 standards, the age at which they were diagnosed, as well as a Family history of relevant illnesses.

The center section from the top;

- Lifetime mood scores - depicted as a sparkline to provide an historical overview of mood swings.

- 14 day detailed overview - provides the detailed scores of mood, sleep, activity and medication from the past 14 days, reported by the patient in the monitoring system, as well as highlight activated triggers.

Medical contact - consist of two sub categories; Last treatment contact, showing when and how the last clinician had contact to the patient, and Last hospitalization, providing an overview of where, when and for how long the patient was hospitalized.

Data analysis - shows the Current and Past Impact Factors, which are the factors that the system calculates as having the biggest influence on the patient's mood state. It also conveys the patient's Adherence rates, which describe how regularly the patient fills out self-assessments, and takes prescribed medicine.

The right side from the top;

Mood forecast - provides the predicted current day mood score and accuracy, as well as a 5-day forecast of the patient's future mood state, based on the collected data from the monitoring system.

Medicine - indicates which medication the patient has been prescribed - both regular and pro necessitate.

- Problem areas - an overview of the problems and focus areas the patient is struggling with in everyday life.

All the content is visible at all times. The information items may have a mouse-over effect, if they can provide you with more detail on the specific item, e.g. hovering over a diagnosis, will provide you with more information on the identity and location of the doctor that made the diagnosis; hovering over one of the items in last treatment contact will provide the journal entry from that day; hovering over an Impact Factor will provide you with the strategies for self-treatment given to the patient. 


\section{FORMATIVE EVALUATION}

For the formative evaluation, we preformed two different types of assessment of the proposed design, based on a paper prototype, as seen in Fig. 1. The prototype contains fictive data, but is modeled according to real patient data.

First, two usability and clinical information systems experts, from the IT University of Copenhagen, performed a heuristic evaluation, which is a usability inspection method for software that helps to identify usability problems early in the user interface design. The evaluators examined the interface and judged its compliance with recognized usability principles - the 'heuristics'. [19].

Secondly, data were collected in the Affective Disorder Clinic at Rigshospitalet in Copenhagen, utilizing the 'Thinkaloud protocol', which has been used successfully in user interface research [20]. This method is used frequently in single user performance evaluations, where the user is asked to voice their thoughts, feelings, and opinions during the evaluation.

A total of 7 clinicians - 3 nurses and 4 doctors participated in the evaluation. All participants were familiar with the MONARCA system. The evaluation followed a detailed script, where the researcher first introduced the project, explained the think aloud procedures, and performed a brief training exercise to familiarize the participants with the concept. Hereafter, the clinician was given the following scenario:

"The time is 13.50, and the last of six previous patients have just left your office. The next patient, whom you have not seen for one and a half months, is scheduled at 14.00. You enter the MONARCA system and select the before mentioned patient in the general overview screen. You are now presented with this new detailed patient overview screen".

The participants then worked through the overview screen, and the researcher elicited more comments based on events that arose during the think aloud protocol. In the end, the participant was asked to rank the different categories according to importance to their work.

All the evaluation sessions were voice recorded, then analyzed using Kvale's first two levels of conversation analysis; self-perception and critical common sense understanding [21].

\section{RESULTS}

From the heuristic evaluation, 4 main comments were provided by the experts; (i) the lifetime mood sparkline does not provide time indicators, (ii) there is inconsistency in headlines of Lifetime mood and 14 days overview, which is not similar to the rest of the categories, (iii) there is too much detailed data in the Patient info category, where details on address, email, and relatives, were supposed to be too detailed for an overview, and finally (iv) the Patient info category could be improved by adding a picture of the patient, as it may support the clinician's recollection. Experts also noted that there was a lot of information in a small amount of space, which can seem overwhelming at first, but they deemed it useful given the goal of providing an overview.
The main outcome of the Think-aloud sessions was that all the clinicians' felt that the design presented " $a$ lot of data..", but all had within the first minute made sense of the different information categories. The general feedback the clinicians provided can be summarized as follows: (i) Time indicators All mentioned the lifetime mood sparkline needed time indicators, as they were not able to make sense of it without. (ii) Orange color - All mentioned that they did not like the orange color used. They said that they would prefer a blue or green nuance instead. (iii) Picture of patients - three of the clinicians mentioned that they would like to have a picture of the patient in the patient info section to help them recall the patients. (iv) Latest journal entry - All the nurses said that it would help their work if the latest entry in the journal were displayed in full text, and not only through a mouse-over function.

During the evaluation, the clinicians mentioned how having this type of overview would provide a much clearer overview, would be time saving, and they foresaw that fewer misunderstandings would occur in subsequent meetings with patients, as sometimes happens when clinicians are not informed of important patient events, e.g. hospitalizations.

When the clinicians were asked to rank the different categories, they were very reluctant to do so, as they found all of them important for the overview. However, the nurses ranked the 14 day detailed overview, the medicine, the patient info, and problem areas as the most important details for their work. The clinicians ranked disease info, 14 day detailed overview, medicine, and medical contact as most relevant.

\section{DISCUSSION}

In the initial design phase, the clinicians were not really interested in getting aid from the system to change or focus the overview screen, based on a systemic interpretation of the patient - supporting comprehension. The clinicians made their own judgments based on the different sources of information, but recognized a great improvement in having all the relevant elements displayed at once - to improve perception. All the clinicians were very enthusiastic about the overview screen during the evaluations, except for one doctor who was not particularly fond of it, as it contained too much information. She knew her patients well and could recall a lot of details about each individual patient, even though there could be long periods between visits. Thus she was only interested in getting the relevant elements from the monitoring system, and not be presented with information she already knew. However, more long-term deployment of our overview solution in clinical settings might reveal cases and situations (e.g., assignment of larger numbers of patients to a doctor) when perception support tools become necessary and can make a difference in the quality of treatment provided.

In their daily work, the nurses are more focused on the subjective side of the treatment, dealing with psychoeducational aspects of a patient's treatment. They would use the screen as a reference point during the conversations with a patient, as it contains all the important items regarding their treatment. Doctors, however, are more interested in objective information, such as a patient's diagnosis, or the medication 
they have been prescribed, and put less emphasis on the particular issues the patients are struggling with in their everyday life. This is also evident from the ranking of categories, where the doctors focused on the diagnoses and prior contact, whereas the nurses looked at problem areas and patient info. Nevertheless, even though they have different focus areas, we found that the given solution would provide support for both, as all the categories included in the overview were found useful for all parts of the treatment.

\section{CONCLUSION}

We have introduced the design and evaluation process of a detailed patient overview screen for supporting the treatment of bipolar disorder patients through the use of a personal monitoring system. Moving through the development phases, we learned that (i) clinicians require a full overview of all patient-critical information on one screen, not hidden within dropdowns or menus, (ii) they are able to use the same overview for different approaches in the treatment, and finally (iii) they do not prefer content to differentiate between patients, diagnoses, or special characteristics. They require system support to improve perception, rather than comprehension processes. All the clinicians were very enthusiastic about the overview screen, and wanted it put to use right away.

The main limitation of this study consists in the fact that the results presented are based on a formative evaluation of our overview screen solution, involving a small group of clinicians and usability experts. However, the collection of these results was important, as they constitute the input to future design and implementation work. Specifically, a clinicians' component of the MONARCA system that will be deployed and further tested in different clinical settings over the next months. From this more complete testing of the whole system we expect to derive further insights on the contribution of the clinicians' overview screen to an improvement of current practice in the treatment of bipolar disorder.

\section{ACKNOWLEDGMENT}

This work has been done in collaboration with a group of clinicians from the Copenhagen Affective Disorder Clinic at the University Hospital of Copenhagen. MONARCA is funded as a STREP project under the FP7 European Framework program. More information can be found at http://monarcaproject.eu/

The final high quality image of the detailed patient overview screen can be downloaded from https://www.dropbox.com/s/cb3ebzkr85tjocz/detailed_patient overview screen.jpg?m

\section{REFERENCES}

[1] L.V. Kessing, P.K. Andersen, P.B. Mortensen, and T.G. Bolwig. Recurrence in affective disorder: I. Case register study. Br J Psychiatry, 172, 1998, 23-28.

[2] R. Morriss, M.A. Faizal, A.P. Jones, P.R. Williamson, C.A. Bolton, J.P. McCarthy. Interventions for helping people recognise early signs of recurrence in bipolar disorder. Cochrane Database of Systematic
Reviews 2007, Issue 1. Art. No.: CD004854.

[3] M. Berk, G.S. Malhi, K. Hallam, C.S. Gama, S. Dodd, A.C. Andreazza, B.N. Frey, and F. Kapczinski. Early intervention in bipolar disorders: Clinical, biochemical and neuroimaging imperatives, Journal of Affective Disorders, Volume 114, Issues 1-3, April 2009, Pages 1-13.

[4] J.M. Bopp, D.J. Miklowitz, G.M. Goodwin, W. Stevens, J. Rendell, and J.R. Geddes. The longitudinal course of bipolar disorder as revealed through weekly text messaging: a feasibility study. Bipolar Disorders, 12: 327-334, 2010.

[5] J. E. Bardram, M. Frost, K. Sz'ant'o, and G. Marcu. The monarca selfassessment system: a persuasive personal monitoring system for bipolar patients, in Proceedings of the 2nd ACM SIGHIT International Health Informatics Symposium, ser. IHI '12. New York, NY, USA: ACM, 2012, pp. 21-30.

[6] E.S. Berner. Clinical decision support systems: State of the Art. AHRQ Publication No. 09-0069-EF. Rockville, Maryland: Agency for Healthcare Research and Quality. June 2009.

[7] L. Mamykina, E. D. Mynatt, and D. R. Kaufman. Investigating health management practices of individuals with diabetes. In Proceedings of the SIGCHI conference on Human Factors in computing systems, CHI '06, pages 927-936, New York, NY, USA, 2006. ACM.

[8] B. K. Smith, J. Frost, M. Albayrak, and R. Sudhakar. Integrating glucometers and digital photography as experience capture tools to enhance patient understanding and communication of diabetes selfmanagement practices. Personal Ubiquitous Comput., 11:273-286, April 2007.

[9] K. A. Siek, K. H. Connelly, Y. Rogers, P. Rohwer, D. Lambert, and J. L. Welch. When do we eat? An evaluation of food items input into an electronic food monitoring application. In Pervasive Health Conference and Workshops, 2006, pages 1-10, 29 2006-dec. 12006.

[10] H.R. Lee, W.R. Panont, B. Plattenburg, J. de la Croix, D. Patharachalam, G. Abowd. Asthmon: Empowering Asthmatic Children's Self-Management with a Virtual Pet. Proc CHI 2010, ACM Press, 2010, pp. 3583-3588.

[11] M.R. Endsley and D.G. Garland (Eds.). (2000). Situation awareness analysis and measurement. Mahwah, NJ: Lawrence Erlbaum

[12] M.R. Endsley. Toward a theory of situation awareness in dynamic systems. Human Factors, 37(1), 1995, 32-64.

[13] V.S. Sorathia (2008). Dynamic Information Management Methodology with Situation Awareness Capability", PhD Thesis, Dhirubhai Ambani Institute of Information and communication Technology (DA-IICT), Gandhinagar, India, 2008.

[14] D. Dorr, L.M. Bonner, A.N. Cohen, R.S. Shoai, R. Perrin, E. Chaney, A.S. Young: Informatics systems to promote improved care for chronic illness: A literature review. J Am Med Inform Assoc 2007, 14(2):156163.

[15] M.H. Trivedi, E.J. Daly, J.K. Kern, B.D. Grannemann, P. Sunderajan and C.A. Claassen. Barriers to implementation of a computerized decision support system for depression: an observational report on lessons learned in "real world" clinical settings. BMC Medical Informatics and Decision Making 2009, 9:6.

[16] A.S. O’Malley, J.M. Grossman, G.R. Cohen, N.M. Kemper, H.H Pham. Are electronic medical records helpful for care coordination? Experiences of physician practices. J Gen Intern Med 2010; 25 (3) 177185.

[17] S.H. Walsh. The clinician's perspective on electronic health records and how they can affect patient care. BMJ 2004;328;1184-1187

[18] American Psychiatric Association. Practice guideline for the treatment of patients with bipolar disorder (revision), American Journal of Psychiatry. 2002;159:1-50

[19] J. Nielsen. Heuristic evaluation. In Nielsen, J., and Mack, R.L. (Eds.), Usability Inspection Methods, John Wiley \& Sons, New York, NY, 1994.

[20] K.A. Ericsson and H.A. Simon,"Verbal reports as data". Psychological Review, Vol. 87, no. 3, pp. 215-251, 1980.

[21] S. Kvale. Interview - An Introduction to Qualitative Research $\begin{array}{lll}\text { Interviewing. } & \text { Sage, } & 1996 .\end{array}$ 